\title{
Con quién hablan los adolescentes mexicanos sobre el SIDA
}

\author{
Cecilia Gayet, MCS, MD, (1) Carolina A. Rosas, MD, (1) \\ Carlos Magis, MSP, ${ }^{(2)}$ Patricia U ribe, MC. ${ }^{(2)}$
}

\section{Gayet C, Rosas CA, Magis C, Uribe P. Con quién hablan los adolescentes mexicanos sobre el SIDA.}

Salud Publica Mex 2002:44:122-128. El texto completo en inglés de este artículo está disponible en: http://www.insp.mx/salud/index.html

\begin{abstract}
Resumen
O bjetivo. Establecer si ciertas características de los jóvenes influyen en la elección de las personas con quienes hablan sobre el SIDA. Material y métodos A partir de una encuesta realizada por el Consejo $\mathrm{N}$ acional para la Prevención y Control del SIDA (CO N ASIDA) en 1997 se hizo una regresión logística multino mial. El estudio abarca a 4886 jóvenes de uno u otro sexo, de 15 a 19 años, en 13 entidades federativas de México. Resultados. El modelo, que incluye las variables sexo, actividad sexual, condición de actividad laboral y escolaridad del padre resultó significativo y altamente predictor de las personas con quienes los jóvenes hablan del SIDA, siempre en comparación con los jóvenes que no hablan con nadie. Los hombres jóvenes hablan del SIDA con su padre más que las mujeres, y las mujeres más con su madre. Los sexualmente activos hablan más con los amigos, y algo menos con los maestros, que los sexualmente inactivos.A mayor escolaridad del padre, los jóvenes tienen más interlocutores y hablan más so bre el SIDA en el ámbito familiar, comparados con los que tienen padre sin escolaridad. Conclusiones. Se sugiere diseñar estrategias diferenciadas de educación en salud sexual de acuerdo con las características de las subpo blaciones. El texto completo en inglés de este artículo está disponible en: http:// www.insp.mx/salud/index.html
\end{abstract}

Palabras clave: adolescencia; síndrome de inmunodeficiencia adquirida; educación sexual; México

\author{
Gayet C, Rosas CA, Magis C, Uribe P. \\ With whom do Mexican \\ teenagers talk about AIDS? \\ Salud Publica Mex 2002;44:122-128. \\ The English version of this paper \\ is available at: http://www.insp.mx/salud/index.html
}

\begin{abstract}
A bstract
Objective. To establish whether certain characteristics of the young influence their choice of people with whom to discuss AIDS. Material and Methods. A national survey was conducted in 1997 by the Consejo N acional para la Prevención y Control del SIDA (CONASIDA, Mexican Council for AIDS Prevention and Control). Study subjects were 4886 male and female 15-19 year-old teenagers. Multinomial logistic regression was used to analyze data. Results. A model including the variables sex, sexual activity, work conditions, and father's schooling level, turned out to be significant and highly predictive of people with whom teenagers discuss AID S, as compared to teenagers speaking with no one. Male teenagers discuss AIDS with their fathers more than female teenagers, and female teenagers discuss AIDS more with their mothers. Sexually active teenagers discuss AIDS more with their friends and less with their teachers than sexually inactive teenagers. The greater schooling level the father has, the more people teenagers have with whom to discuss AIDS and the more they discuss AIDS at home, compared to teenagers with fathers without schooling. Conclusions. Differentiated sexual education training strategies should be designed in accordance with subpopulations' character istics. The English version of this paper is available at:http://www.insp.mx/salud/ index.html
\end{abstract}

key words: adolescence; acquired immunodeficiency syndrome; sex education; Mexico

(1) El Colegio de México, México, D.F., México.

(2) Centro N acional para la Prevención y el Control del SIDA (CEN SIDA), México, D.F., México.

Fecha de recibido: 8 de enero de 2001 • Fecha de aprobado: 11 de octubre de 2001

Solicitud de sobretiros: Cecilia Gayet. El Colegio de México, CED DU. Carretera al Ajusco 20. Colonia Pedregal de Santa Teresa. 01000 México, D.F. Correo electrónico: cgayet@ colmex.mx 
E $n$ el inicio del siglo XXI permanecen interrogantes que requieren más y nuevas investigaciones. Entre ellos, la conducta sexual de los adolescentes en un mundo con considerables riesgos para la salud, deviene objeto de relevancia política y científica. Una preocupación para los investigadores y las autoridades es cómo brindar conocimientos a los jóvenes que les permitan prevenir embarazos no deseados e infecciones de transmisión sexual, entre ellas el SIDA.

Con el objetivo de diseñar programas efectivos de educación sexual se realizaron varias investigaciones para determinar cuáles son los canales de información que más utilizan los jóvenes en materia de sexualidad. Se ha hecho énfasis en las redes de comunicación sobre temas específicos como embarazo, anticoncepción, masturbación, SIDA/infecciones de transmisión sexual, noviazgo, etcétera.

En el contexto de estudios regionales, Ibáñez y Odriozola ${ }^{1}$ hicieron una encuesta entre jóvenes universitarios de Puebla. En esa investigación interesaba conocer las fuentes de educación sexual usadas, según temas y sexo de los entrevistados. Las fuentes más importantes fueron los amigos y los padres para las mujeres, en tanto que para los hombres lo fueron los amigos y los libros. Otro estudio, realizado en Aguascalientes, encontró que "salud sexual" es el tema preferido sobre el que los adolescentes (entre 14 y 22 años) de escuelas de nivel medio superior desearían conocer más, en el área de educación sexual. ${ }^{2}$ Los varones declararon acudir a resolver sus dudas sobre sexualidad principalmente con los amigos, seguidos casi en el mismo nivel por el maestro, el padre y la madre. Las mujeres, en cambio, prefirieron acudir en primer lugar con la madre, y en segundo lugar con los amigos.

En el ámbito nacional, el Consejo Nacional de Población (Conapo) realizó la Encuesta Nacional sobre Sexualidad y Familia en Jóvenes de Educación Media Superior. ${ }^{3}$ Los resultados de esta encuesta indican que entre los canales que más proporcionaban información sobre sexualidad los varones refirieron a los maestros, los amigos y el padre, en tanto que las mujeres mencionaron a la madre, las maestras y las amigas. Conforme aumentaba la edad de los entrevistados, la familia iba dejando de ser un interlocutor importante, incrementándose la presencia de los médicos y los libros. Más recientemente, la Fundación Mexicana para la Planeación Familiar aplicó una encuesta a adolescentes de entre 13 y 19 años de edad en sus zonas de influencia. ${ }^{4}$ Una de las preguntas se refería a la persona preferida para que les hablara sobre el SIDA. Los médicos fueron mencionados en primer lugar tanto por varones como por mujeres, seguidos por los maestros. Las mujeres consideraron a los padres en un nivel de importancia muy semejante al de los maestros, a diferencia de los varones, entre los cuales la preferencia por los padres fue menor. En esta encuesta, los amigos no aparecen como fuente deseada de información sobre SIDA.

Buena parte de las diferencias entre los resultados de investigación pueden deberse a que las preguntas formuladas en las distintas encuestas no eran semejantes. Así, hablar sobre el SIDA remite a situaciones de interacción entre los participantes del acto de habla y puede incluir la comunicación de experiencias personales. En cambio, recibir información sobre el SIDA tiene una connotación más estática, donde los componentes parecen ser un "transmisor" capacitado y un receptor pasivo. En situaciones de este último tipo, se privilegian los conocimientos científicos sobre el tema más que el intercambio sobre experiencias personales. Por esta razón, las respuestas de los jóvenes pueden ser totalmente distintas según cómo se haga la pregunta. Los interlocutores de "hablar sobre SIDA" no necesariamente son los mismos que los "informantes sobre SIDA".

Sin embargo, los resultados dispares arrojados por las investigaciones trajeron la discusión sobre la pertinencia de enfocar los programas de educación sexual que se llevaban a cabo fuera del ámbito escolar hacia acciones entre pares (de joven a joven) o hacia la comunicación en el seno de la familia (de padres a hijos). Hubo un acuerdo general sobre el hecho de que los programas de educación sexual dentro del ámbito escolar debían fortalecerse, y la polémica se situó sobre qué tipo de programas privilegiar fuera de la escuela con los escasos recursos de las organizaciones no gubernamentales dedicadas al tema. ${ }^{5}$ En principio, se acepta que en un escenario de recursos económicos no limitados, todo tipo de acciones que promuevan las diversas formas de comunicación sobre sexualidad pueden ser válidas para proporcionar información a la población joven. Pero en el contexto de recursos limitados, la forma en que se dirima este interrogante tendrá consecuencias sobre las acciones diseñadas por las organizaciones no gubernamentales, ya sea educando a líderes adolescentes como promotores o capacitando a los padres para transmitir este tipo de información.

En el marco de esta discusión, se hace este trabajo que tiene por objetivo tratar de establecer si ciertas características de los jóvenes influyen en la elección de las personas con quienes hablan del SIDA, a partir de una encuesta del Consejo Nacional para la Prevención y Control del SIDA (CONASIDA) realizada en 1997, utilizando como técnica la regresión logística multinomial. 


\section{Material y métodos}

En 1997, el CONASIDA aplicó una encuesta a adolescentes entre 15 y 19 años de edad, padres y maestros, cuyo objetivo fue evaluar la campaña para la prevención del SIDA, desarrollada por esa institución en los medios de comunicación masiva en el Distrito Federal, Estado de México, Veracruz, Morelos, Baja California, Baja California Sur, Nuevo León, Durango, Jalisco, Guerrero, Chiapas, Quintana Roo y Yucatán. ${ }^{6}$

En esta investigación sólo consideramos la encuesta aplicada a los adolescentes, cuyo número de entrevistados fue de 7393 (52\% fueron hombres y $48 \%$ mujeres), escolares (68\%) y no escolares (32\%). Para elegir a los adolescentes escolarizados se hizo una selección sistemática de escuelas de nivel medio superior y se utilizó un muestreo estratificado, polietápico, de conglomerados. Los adolescentes no escolarizados de las zonas urbanas fueron encontrados en sitios de gran afluencia alrededor de las escuelas escogidas (estaciones del metro, parques y paradas de camión), y los de las zonas rurales, en las plazas. El muestreo utilizado para los adolescentes no escolarizados fue polietápico, similar al que se utilizó para seleccionar las escuelas. Los puntos de afluencia de población no escolarizada fueron definidos en las zonas sorteadas, a la vez que se establecieron cuotas por puntos de afluencia. Si bien la sub-muestra de adolescentes no escolarizados no fue seleccionada de manera aleatoria, se la incluyó en el análisis a fin de contar con un universo más heterogéneo.

Entre las preguntas figuraba "¿con quién has platicado sobre SIDA (contagio, prevención, uso del condón)?", y las respuestas posibles eran: "1) padre, 2) madre, 3) maestro, 4) amigos y 5) con nadie". De estas respuestas, desde la 1 a la 4 no eran mutuamente excluyentes, por lo que se cuenta con una gran combinación de las mismas. Las respuestas a esta pregunta se utilizaron como base del análisis.

A partir de las respuestas a la pregunta " ¿con quién has platicado sobre SIDA (contagio, prevención, uso del condón)?", construimos la variable dependiente del modelo de regresión multinomial. Como señalamos anteriormente, dado que las respuestas 1 a 4 no eran mutuamente excluyentes, creamos una variable cuyas categorías eran cada una de las posibles combinaciones entre estas cuatro respuestas, además de la 5 (con nadie) que excluía a las restantes.

En segundo lugar, hicimos una selección de las combinaciones de la variable que consideramos relevantes para nuestro estudio, tomando en consideración la literatura sobre el tema. Elegimos las siguientes opciones como categorías de la variable dependiente "personas con quienes hablan los adolescentes sobre SIDA": 1. Madre, 2. Padre, 3. Maestro, 4. Amigos, 5. Madre y padre, 6. Maestro y amigos, 7. Madre, padre, maestro y amigos, 8 . Con nadie. Las primeras cuatro son las opciones simples, y dentro de las combinaciones seleccionamos el ámbito familiar (categoría 5), el ámbito extra-familiar (categoría 6), y la combinación de todos los interlocutores posibles (categoría 7).

De los 7393 adolescentes entrevistados, hemos considerado una submuestra de 4886 (hombres y mujeres), en función de las categorías de la variable dependiente que eran de nuestro interés.

Consideramos que la selección del interlocutor para hablar del SIDA depende de ciertas características de los adolescentes. Así, el sexo, el nivel social al que pertenecen, la condición de actividad sexual y la condición de actividad laboral influirían en la preferencia del interlocutor. De manera más específica, supusimos que el sexo del adolescente (hombre o mujer) puede diferenciar a la persona con la que prefieren hablar, como sugirieron las investigaciones reseñadas. Supusimos que las mujeres tienden a hablar más con sus madres y los varones con sus padres y amigos. Asimismo, que los sexualmente activos tenderían a platicar menos con los adultos que con el grupo de pares, dado que la sexualidad en la adolescencia parece ser un tema tabú y es posible que sientan que deben ocultar esta actividad, principalmente las mujeres. Respecto a la condición de actividad laboral del adolescente, supusimos que aquellos que están insertos en ámbitos laborales tienen un acceso diferente (menor) a la temática, y la plática con los adultos se dificulta por la misma razón. Por otra parte, consideramos necesario controlar la escolaridad del padre como aproximación al nivel social familiar del adolescente. Supusimos que en aquellos hogares donde el padre alcanzó altos niveles de escolaridad existe mayor apertura para hablar sobre el SIDA.

No se incluyó la variable edad dado que, en primer lugar, el universo es bastante homogéneo respecto a esta variable (sólo entre 15 y 19 años) y, en segundo lugar, se correlaciona con actividad sexual (a mayor edad, mayor probabilidad de ser sexualmente activos). Por otro lado, estimamos más importante que la edad el hecho de que fueran o no sexualmente activos para comunicarse con otras personas respecto a temas que se relacionan con la sexualidad.

La regresión logística multinomial es útil para situaciones en las que se desea clasificar sujetos con base en valores de un conjunto de variables predictivas. Es similar a la regresión logística, pero es más general porque la variable dependiente no está restringida a sólo dos categorías. La variable dependiente deberá ser 
categórica, en tanto que las explicativas pueden ser factores o covariables. La especificación de las variables del modelo generado puede verse en el cuadro I. En este tipo de regresión, los resultados se establecen en función de una categoría de comparación. En nuestro caso, seleccionamos como categoría de comparación para la variable dependiente a la opción hablar "con nadie". La gran ventaja de este tipo de técnica estadística es que permite conocer el impacto de cada una de las variables controlando las restantes, y los efectos de las interacciones entre ellas. Permite, asimismo, mostrar de manera sintética las asociaciones entre variables y la calidad del modelo en su conjunto. Los datos fueron procesados con el paquete estadístico SPSS 9.0 para Windows.

La distribución de los 4886 casos seleccionados según la variable dependiente y las variables explicativas consideradas muestra que $52.2 \%$ de los entrevistados eran hombres y $47.8 \%$ mujeres. Del total de jóvenes de uno $\mathrm{u}$ otro sexo, $33.6 \%$ eran sexualmente activos, y $68.8 \%$ eran estudiantes y no trabajaban. Un $5.4 \%$ de los entrevistados tenía padre sin escolaridad, $36.7 \%$ tenía padre con primaria completa, $40.9 \%$ tenía padre con nivel medio completo y $17 \%$ tenía padre con estudios de nivel superior completo (cuadro II).

\section{Resultados}

El modelo de regresión logística multinomial resultó significativo en su conjunto $(p<0.05)$, y un buen predictor, según las pruebas de función de verosimilitud, de bondad de ajuste, del coeficiente de Nagelkerke y la comparación entre valores observados y esperados. Las variables sexo y escolaridad del padre pesan más en la verosimilitud del modelo que las variables actividad sexual y condición de actividad (cuadro III).

\section{Cuadro II \\ Frecuencia de los casos según las variables del MODELo, MÉXICO, 1997}

\begin{tabular}{lrr}
$\begin{array}{l}\text { Variables independientes } \\
\text { Sexo }\end{array}$ & Frecuencia & Porcentaje \\
Hombres & & \\
\hline Mujeres & 2552 & 52.2 \\
\hline Total & 2334 & 47.8 \\
$\quad 4886$ & 100.0 \\
$\begin{array}{l}\text { Condición de actividad sexual } \\
\quad \text { Sexualmente activos }\end{array}$ & 1641 & \\
\hline$\quad$ Sexualmente inactivos & 3245 & 66.6 \\
\hline$\quad$ Total & 4886 & 100.0 \\
$\quad$ & & \\
Condición de actividad laboral & & \\
N o trabaja & 3364 & 68.8 \\
\hline Trabaja & 1522 & 31.2 \\
\hline Total & 4886 & 100.0
\end{tabular}

\begin{tabular}{lrr}
$\begin{array}{l}\text { Escolaridad del padre } \\
\text { Primaria completa }\end{array}$ & 1795 & 36.7 \\
\hline N ivel medio (completo) & 1996 & 40.9 \\
\hline N ivel superior (completo) & 830 & 17.0 \\
\hline Sin estudios & 265 & 5.4 \\
\hline Total & 4886 & 100.0
\end{tabular}

Variable independientes

Personas con quienes hablan los adolescentes sobre el SIDA

\begin{tabular}{lrr} 
Padre & 244 & 5.0 \\
\hline Madre & 562 & 11.5 \\
\hline Maestro & 902 & 18.5 \\
\hline A migo & 1440 & 29.5 \\
\hline Padre y madre & 217 & 4.4 \\
\hline Maestro y amigo & 345 & 7.1 \\
\hline Madre, padre, maestro y amigos (todos) & 408 & 8.4 \\
\hline Nadie & 768 & 15.7 \\
\hline Total & 4886 & 100.0
\end{tabular}

Cuadro I

Modelo de regresión logística multinomial generado, México, 1997

Variable dependiente Personas con quienes hablan los adolescentes sobre el SIDA



\section{Variction}

ariables independientes

Condición de
actividad laboral

1. No trabaja 2. Trabaja*
1. Sexualmente activos

1. Hombre 2. Mujer*

2. Sexualmente inactivos*

(1)

3. Maestro

4. Amigos

5. Madre y padre

6. Maestro y amigos

7. Madre, padre, maestro y amigos

8. Con nadie*

* Categorías de comparación 
Entre los principales resultados advertimos que algunas de las hipótesis parecen confirmarse (en el cuadro IV pueden verse los coeficientes de la regresión y los niveles de significación). El sexo del entrevistado es significativo $(p<0.05)$ para quienes hablan con el "padre", la "madre" o con "todos", en comparación con los que no hablan "con nadie", y no lo es para las otras categorías de la variable dependiente. Así, los hombres hablan 4.7 veces más sobre el SIDA con su padre que las mujeres, y las mujeres hablan con su madre 3.2 veces más que los hombres, en comparación con aquellos que no hablan con nadie. Todos los resul-

\section{Cuadro III \\ Peso diferencial de las variables SEGÚN LOS TESTS DE RAZÓN DE VEROSIMILITUD (EFEctos PRINCIPALes). MéXICo, 1997}

\begin{tabular}{lrrrr} 
Efecto & $\begin{array}{l}-2 \text { Log verosimilitud } \\
\text { del modelo reducido }\end{array}$ & $\begin{array}{c}\text { Ji } \\
\text { cuadrada }\end{array}$ & $\begin{array}{c}\text { Grados de } \\
\text { libertad }\end{array}$ & Sig. \\
& 986.7759 & 0.0000 & \multicolumn{1}{c}{0} & - \\
\hline Intersección & 1041.3400 & 54.5641 & 7 & 0.0000 \\
\hline Condición de actividad laboral & 1246.2148 & 259.4389 & 21 & 0.0000 \\
\hline Escolaridad del padre & 1247.2355 & 260.4597 & 7 & 0.0000 \\
\hline Sexo & 1067.3312 & 80.5554 & 7 & 0.0000 \\
\hline Condición de actividad sexual & & & &
\end{tabular}

Cuadro IV

Modelo de regresión logística multinomial. México, 1997

\begin{tabular}{|c|c|c|c|c|c|c|}
\hline \multirow{2}{*}{\multicolumn{2}{|c|}{$\begin{array}{l}\text { Personas con quienes hablan } \\
\text { los adolescentes sobre el SIDA }\end{array}$}} & \multirow[b]{2}{*}{ Beta } & \multirow[b]{2}{*}{ Exp(Beta) } & \multirow{2}{*}{$\underset{(p<0.05)}{\text { Sig. }}$} & \multicolumn{2}{|c|}{ 95\% Intervalo de confianza para $\operatorname{Exp}(B)$} \\
\hline & & & & & Límite inferior & Limite superior \\
\hline \multirow[t]{6}{*}{ Padre } & Condición de actividad laboral=no trabaja & 0.54 & 1.71 & 0.0014 & 1.23 & 2.38 \\
\hline & Escolaridad del padre=primaria & 0.95 & 2.59 & 0.0759 & 0.91 & 7.41 \\
\hline & Escolaridad del padre=nivel medio & 1.94 & 6.98 & 0.0002 & 2.48 & 19.63 \\
\hline & Escolaridad del padre=nivel superior & 2.80 & 16.44 & 0.0000 & 5.66 & 47.73 \\
\hline & Sexo=hombre & 1.55 & 4.71 & 0.0000 & 3.18 & 6.98 \\
\hline & Condición de actividad sexual=activos & 0.08 & 1.08 & 0.6308 & 0.79 & 1.47 \\
\hline \multirow[t]{6}{*}{ Madre } & Condición de actividad laboral=no trabaja & 0.74 & 2.09 & 0.0000 & 1.61 & 2.72 \\
\hline & Escolaridad del padre=primaria & 0.20 & 1.22 & 0.4172 & 0.75 & 1.97 \\
\hline & Escolaridad del padre=nivel medio & 0.94 & 2.56 & 0.0001 & 1.59 & 4.12 \\
\hline & Escolaridad del padre=nivel superior & 1.34 & 3.84 & 0.0000 & 2.24 & 6.57 \\
\hline & Sexo=hombre & -1.15 & 0.32 & 0.0000 & 0.25 & 0.40 \\
\hline & Condición de actividad sexual=activos & -0.11 & 0.90 & 0.4215 & 0.69 & 1.17 \\
\hline \multirow[t]{6}{*}{ Maestro } & Condición de actividad laboral=no trabaja & 0.55 & 1.74 & 0.0000 & 1.41 & 2.15 \\
\hline & Escolaridad del padre=primaria & 0.46 & 1.59 & 0.0177 & 1.08 & 2.33 \\
\hline & Escolaridad del padre=nivel medio & 0.51 & 1.66 & 0.0110 & 1.12 & 2.45 \\
\hline & Escolaridad del padre=nivel superior & 0.80 & 2.23 & 0.0007 & 1.40 & 3.55 \\
\hline & Sexo=hombre & 0.13 & 1.14 & 0.1974 & 0.93 & 1.40 \\
\hline & Condición de actividad sexual=activos & -0.53 & 0.59 & 0.0000 & 0.47 & 0.73 \\
\hline \multirow[t]{6}{*}{ Amigo } & Condición de actividad laboral=no trabaja & 0.23 & 1.25 & 0.0178 & 1.04 & 1.51 \\
\hline & Escolaridad del padre=primaria & 0.50 & 1.65 & 0.0044 & 1.17 & 2.32 \\
\hline & Escolaridad del padre=nivel medio & 0.71 & 2.03 & 0.0001 & 1.43 & 2.88 \\
\hline & Escolaridad del padre=nivel superior & 1.10 & 3.00 & 0.0000 & 1.97 & 4.55 \\
\hline & Sexo=hombre & -0.02 & 0.98 & 0.8254 & 0.81 & 1.18 \\
\hline & Condición de actividad sexual=activos & 0.29 & 1.34 & 0.0028 & 1.11 & 1.62 \\
\hline \multirow[t]{6}{*}{ Padre y madre } & Condición de actividad laboral=no trabaja & 0.44 & 1.56 & 0.0117 & 1.10 & 2.20 \\
\hline & Escolaridad del padre=primaria & 1.57 & 4.82 & 0.0320 & 1.14 & 20.32 \\
\hline & Escolaridad del padre=nivel medio & 2.72 & 15.17 & 0.0002 & 3.66 & 62.89 \\
\hline & Escolaridad del padre=nivel superior & 3.31 & 27.27 & 0.0000 & 6.39 & 116.32 \\
\hline & Sexo=hombre & 0.01 & 1.01 & 0.9393 & 0.73 & 1.40 \\
\hline & Condición de actividad sexual=activos & 0.22 & 1.25 & 0.1920 & 0.90 & 1.73 \\
\hline \multirow[t]{6}{*}{ Maestro y amigo } & Condición de actividad laboral=no trabaja & 0.25 & 1.28 & 0.0776 & 0.97 & 1.70 \\
\hline & Escolaridad del padre=primaria & 0.44 & 1.56 & 0.1258 & 0.88 & 2.74 \\
\hline & Escolaridad del padre=nivel medio & 0.91 & 2.49 & 0.0015 & 1.42 & 4.37 \\
\hline & Escolaridad del padre=nivel superior & 1.20 & 3.30 & 0.0002 & 1.74 & 6.26 \\
\hline & Sexo=hombre & -0.17 & 0.84 & 0.2133 & 0.65 & 1.10 \\
\hline & Condición de actividad sexual=activos & -0.16 & 0.85 & 0.2676 & 0.64 & 1.13 \\
\hline \multirow[t]{6}{*}{ Todos } & Condición de actividad laboral=no trabaja & 0.68 & 1.98 & 0.0000 & 1.48 & 2.64 \\
\hline & Escolaridad del padre=primaria & 1.12 & 3.06 & 0.0066 & 1.37 & 6.86 \\
\hline & Escolaridad del padre=nivel medio & 1.90 & 6.69 & 0.0000 & 3.01 & 14.89 \\
\hline & Escolaridad del padre=nivel superior & 2.79 & 16.33 & 0.0000 & 7.10 & 37.56 \\
\hline & Sexo=hombre & -0.42 & 0.66 & 0.0014 & 0.51 & 0.85 \\
\hline & Condición de actividad sexual=activos & -0.21 & 0.81 & 0.1387 & 0.61 & 1.07 \\
\hline
\end{tabular}


tados deben considerarse manteniendo el resto de las variables constante.

Respecto a la actividad sexual, ser sexualmente activo sólo es significativo $(p<0.05)$ cuando los adolescentes platican con amigos o con el maestro, y no es significativo para los adolescentes que declaran hablar sobre el SIDA con el padre, con la madre, o con ambos, comparados con aquellos que no hablan con nadie. Los sexualmente activos hablan 1.3 veces más con los amigos que los sexualmente inactivos $\mathrm{y}$, a su vez, los inactivos hablan 1.7 veces más con sus maestros que los activos, en comparación con los que no hablan con nadie, lo que podría ser indicativo de que los sexualmente activos hablan menos con los adultos que con los pares, aunque debe tenerse en cuenta que los valores son pequeños.

Habíamos postulado la hipótesis de que los que trabajan hablan menos con los adultos sobre el SIDA que los que no trabajan, comparados con los que no hablan con nadie, y esto parece verificarse. En términos generales, los que no trabajan hablan más tanto con adultos como con pares que los que trabajan, en comparación con los que no hablan con nadie. Y la magnitud parece ser mayor con los adultos (padre: 1.7 veces más, madre: 2.1 veces más, maestro: 1.7 veces más) que con los amigos (1.2 veces más).

Otro supuesto era que a mayor escolaridad del padre, los adolescentes hablaban más en el ámbito familiar (padre, madre y padre-madre). Lo que se verifica es que a mayor escolaridad del padre, los jóvenes hablan más con todas las categorías de la variable dependiente, respecto de los que no hablan con nadie y a los padres que no tienen escolaridad. Pero hablan aún más con el padre y con el padre y la madre en conjunto, a medida que aumenta la escolaridad del padre. Así, la categoría con valor más alto corresponde a los adolescentes con padre que tiene nivel superior completo.

El ámbito familiar, representado en esta investigación por padres y madres, se constituyó en el escenario privilegiado por los adolescentes que no trabajan, son sexualmente inactivos y tienen padres que han alcanzado mayor escolaridad, para platicar sobre contagio o prevención del SIDA, el uso del condón, etcétera. Si bien, en términos generales, estos adolescentes manifestaron que hablan sobre el SIDA en el ámbito familiar, hay que recordar que el sexo de los jóvenes condicionó la elección del interlocutor, en la medida en que los hijos prefirieron platicar con el padre y las hijas con las madres.

Por otra parte, teniendo en cuenta el ámbito extra-familiar, pudimos observar que los sexualmente activos son quienes reconocen a los amigos y a los maestros (en ese orden de importancia) como sus interlocutores para hablar sobre el SIDA. La actividad sexual parece propiciar un mayor acercamiento entre pares, relegando el ámbito familiar a un segundo lugar.

\section{Discusión}

Los programas de educación sexual permiten que disminuyan ciertos riesgos que pueden devenir una práctica sexual desprotegida, ya que se transmite información, se alienta el uso del condón y se hace énfasis en la dimensión placentera de la sexualidad. ${ }^{7}$ En la formación y modificación de actitudes y conductas de los jóvenes, la comunicación (con padres, amigos, etcétera) juega un papel fundamental. Por esto, una estrategia para enfrentar al SIDA es la prevención y la difusión de información a través del desarrollo de programas y políticas educativas.

Sin embargo, también se ha demostrado que una educación sexual que no se adecue a los contextos socioculturales en los que se desarrollan los jóvenes es poco efectiva y no necesariamente da lugar a cambios en las prácticas riesgosas. Así, la investigación ocupa un lugar importante al poner de manifiesto las particularidades y las necesidades poblacionales en materia de educación sexual, porque no es eficiente diseñar estrategias que no se correspondan con dichas necesidades. $^{8}$

En este sentido, los resultados de este análisis sugieren que no es conveniente proponer acciones para todos los adolescentes en conjunto, sin tener en cuenta sus particularidades. El nivel social al que pertenecen (al que nos aproximamos en este caso con la escolaridad del padre), su condición de actividad sexual y laboral, y el sexo, parecen determinar la elección del interlocutor para hablar del SIDA. Sin embargo, la generalización de estos resultados debe hacerse con cautela, dado que la muestra no fue totalmente aleatoria.

En la medida en que consideramos que una estrategia de educación sexual eficiente debe escuchar a sus destinatarios, poner atención sobre los interlocutores señalados por los adolescentes es una tarea fundamental. Por un lado, para el grupo de adolescentes que no ha comenzado su actividad sexual y proviene de niveles sociales favorecidos (se pueden permitir no trabajar y sus padres alcanzaron altos niveles de escolaridad), parece adecuarse una estrategia de educación sexual en la cual se capacite a los padres, ya que fueron los interlocutores privilegiados por estos adolescentes. Por otro lado, al grupo de adolescentes que ya ha iniciado su actividad sexual, debería corresponderle una estrategia de educación sexual en la cual la información y las dudas se dirimieran entre pares. Este 
último grupo es el que más interesa desde el punto de vista de la prevención del SIDA, ya que las prácticas sexuales desprotegidas en las que pudieran incurrir constituyen un riesgo importante de contraer la enfermedad. Para estos adolescentes no tendría demasiada efectividad una educación sexual de padres a hijos, ya que no hablan sobre el tema en el ámbito familiar.

En síntesis, si se desea tomar en consideración los interlocutores actuales que tienen los adolescentes como base para diseñar programas, hay que pensar en estrategias diferenciadas de acuerdo con las características de las subpoblaciones. Un posible criterio para diferenciar las estrategias de educación sexual sería considerar el nivel social de pertenencia. Para aquellos de segmentos altos, parecerían ser más adecuadas las acciones dirigidas a los padres, a fin de que les transmitan información a sus hijos. Pero en segmentos sociales desfavorecidos, donde los padres no aparecen como interlocutores privilegiados, tal vez una estrategia de capacitación entre jóvenes sería más efectiva (entre pares o de joven a joven).

\section{Agradecimientos}

Los autores agradecen al profesor Fernando Cortés, de El Colegio de México, por sus enseñanzas y comentarios tanto en aspectos estadísticos como en los teórico-metodológicos, y a la Dra. Fátima Juárez, de la London School of Hygiene and Tropical Medicine, por la transmisión de su valiosa experiencia en el tema de salud sexual y reproductiva de los adolescentes.

\section{Referencias}

1. Ibáñez-Brambila B, 0 driozola-U rbina A. Educación sexual en estudiantes universitarios. Psicol Soc Mex 1992;4:72-82.

2. Meza-Muñoz G, Muñoz-Gutiérrez AM, Reyes-Rodríguez A. Sexualidad adolescente.A guascalientes: Gobierno del Estado de Aguascalientes, 1995; Cuadernos de Trabajo, D esarrollo Social, N 0. 32, noviembre-diciembre.

3. Consejo $\mathrm{N}$ acional de Población. Encuesta $\mathrm{N}$ acional sobre Sexualidad y Familia en Jóvenes de Educación Media Superior. México, D.F.: C O N A PO, 1988.

4. Fundación Mexicana para la Planeación Familiar, AC. (MEXFAM). Encuesta Gente Joven 1999. México, D.F: MEX FAM, 1999.

5. Juárez F, G ayet $C$. Safe passages to adulthood: Enabling young peo ple to improve their sexual and reproductive health. Contextual Situational Analysis: Mexico. Brighton: University of Southampton \& University of London, 2000.

6. Secretaría de Salud / CO N A SID A / Project C oncern International. Campaña informativa de prevención delVIH/SIDA, Fase III, Encuesta de opinión de adolescentes entre 15 y 19 años de edad. México, D.F.: SSA, 1998.

7. Pick de W eiss S, Givaudan M, Saldivar-G arduño A. La importancia de los factores psicosociales en la educación sexual de los adolescentes. Perinatol Reprod Hum 1996;10(2):143-150.

8 Torres MA. Comportamiento erótico de los y las adolescentes. Arch Hispanoam Sexolo 1998; 4(2):259-306. 